\title{
Non-Odontogenic Toothache Caused by the Fungal Ball of Maxillary Sinus: Case Reports
}

\author{
Ji-Woo Ha ${ }^{1}$, Won Jung ${ }^{2}$, Kyung-Eun Lee ${ }^{2}$, Bong-Jik Suh ${ }^{2}$ \\ 'Department of Oral Medicine, School of Dentistry, Jeonbuk National University, Jeonju, Korea \\ ${ }^{2}$ Department of Oral Medicine, Institute of Oral Bioscience, School of Dentistry, Jeonbuk National University, Jeonju, Korea
}

Received November 23, 2019

Revised December 10, 2019

Accepted December 10, 2019

\section{Correspondence to:}

Bong-Jik Suh

Department of Oral Medicine, School of

Dentistry, Jeonbuk National University, 567

Baekje-daero, Deokjin-gu, Jeonju 54896,

Korea

Tel: +82-63-250-2060

Fax: +82-63-250-2058

E-mail: yonam@jbnu.ac.kr

https://orcid.org/0000-0002-1817-4645
A fungal ball $(\mathrm{FB})$ of the paranasal sinuses is a chronic, non-invasive fungal sinusitis defined as the accumulation of dense aggregation of fungal hyphae in a sinus cavity. A patient with FB infection in a sinus cavity has usually non-specific symptoms such as post-nasal drip, nasal congestion, headache. However, facial pain and toothache can be developed if FB infection is in maxillary sinus. The aim of this case report is to present two cases of FB of the maxillary sinus which caused toothache in the upper molar region. It is also to make dental practitioners consider the non-odontogenic origins of toothache and to pay special attention to avoid unnecessary dental treatment.

Key Words: Chronic sinusitis; Fungal ball; Non-odontogenic toothache

\section{INTRODUCTION}

Orofacial pain can be a common clinical problem [1]. A total of $42 \%$ of the general population experienced one or more types of orofacial pain in the past 6 months. Toothache was the most common pain which was reported by $26.8 \%$ of the population experienced orofacial pain [2]. However, orofacial pain can be a diagnostic dilemma for dental practitioners in clinical practice because most of the pain in the orofacial region mimics odontogenic pain and sometimes the site of pain might not be coincident with the origin of pain. In these regards, appropriate diagnosis and management were challenges for dentists $[1,3]$.

Most toothaches are originated from pulpal or adjacent periodontal tissues. The orofacial pain from dental origin was termed "odontogenic toothache" [4]. "Non-odontogenic toothache" defined as the pain which is perceived on pulpal and adjacent structure but not originated from the tooth or periodontal tissues [4]. If clinical and radiographic examinations did not reveal any pathologic findings in teeth or periodontal structures, nonodontogenic origin should be considered [5,6]. Nonodontogenic toothache can be caused by a variety of causes such as muscle disorders, sinus and nasal mucosal problems, neuropathic pain, neurovascular pain, psychogenic problem, or cardiogenic disease [5]. According to Linn et al. [7], 44 out of 100 patients who suffered from non-odontogenic orofacial pain had either tooth extraction or root canal treatment in an attempt to resolve their pain. If the origin of toothache was misdiagnosed, unnecessary dental treatment such as root canal treatment or extractions could be performed and the patient's pain would not be alleviated through such treatment. Therefore, the origin of toothache should be found correctly before dental treatment is performed [5]. Further tests and consultation with other medical specialties have to be performed to find the origin of a toothache.

The objective of this case report is to present uncommon cases of two patients who had the nonodontogenic toothache caused by fungal ball (FB) of the maxillary sinus. 


\section{CASE REPORT}

\section{Case 1}

A 41-year-old female referred from the department of conservative dentistry at the Jeonbuk National University Hospital with a chief complaint of persisted toothache on her maxillary left molar area. Her past history revealed that her toothache started about two years ago. She visited a dental clinic and dental caries was found on the first and second premolar of left upper dentition.

Although root canal treatment was done in a dental clinic, the symptoms were not improved. She visited the department of conservative dentistry for the surgical treatment on the first and second premolar of left maxillary dentition. However, toothache on the molar area was not resolved although apicoectomy was finished.

Clinical examination was performed and findings were including tenderness to palpation on multiple sites with both sides of face, neck, and shoulder. There was no mouth opening limitation. Panoramic and periapical radiograph showed no specific findings (Fig. 1).

She was diagnosed with myofascial pain with referral and toothache was considered as referred pain from myofascial pain. The patient was treated with conservative treatment including patient education, physical therapy, and oral appliance. The symptoms of myofascial pain had been improved about a year later, but toothache had been remained. Other reasons for toothache should be considered.
Cone beam computed tomography (CBCT) taken before she visited our department, revealed moderate mucosal thickening including small calcified bodies of the left maxillary sinus (Fig. 2). She was referred to the department of otorhinolaryngology for the treatment of the left maxillary sinus. It was diagnosed with FB from endoscopic features and histopathological findings after surgical removal under general anesthesia. Her toothache was disappeared about two months later.

\section{Case 2}

A 62-year-old female with a chief complaint of toothache in the right upper molar area was referred to our department from the department of conservative dentistry.

She had toothache in the right upper molar area about five months ago. Root canal re-treatment was treated on first premolar, second premolar and first molar of right

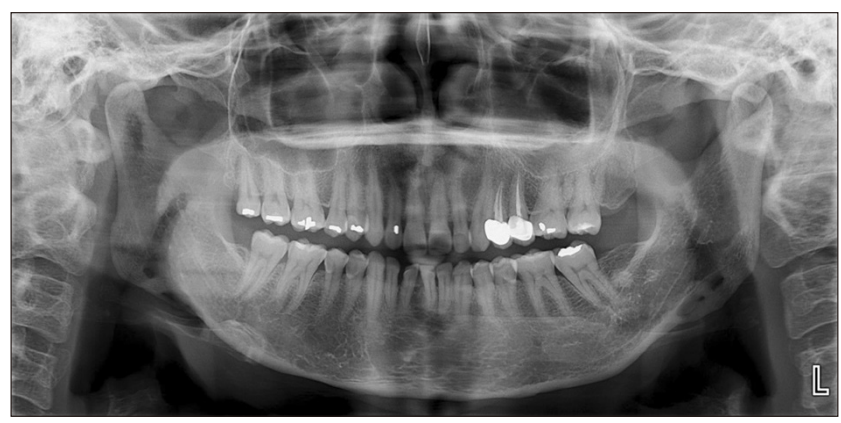

Fig. 1. Panoramic view shows no remarkable findings.
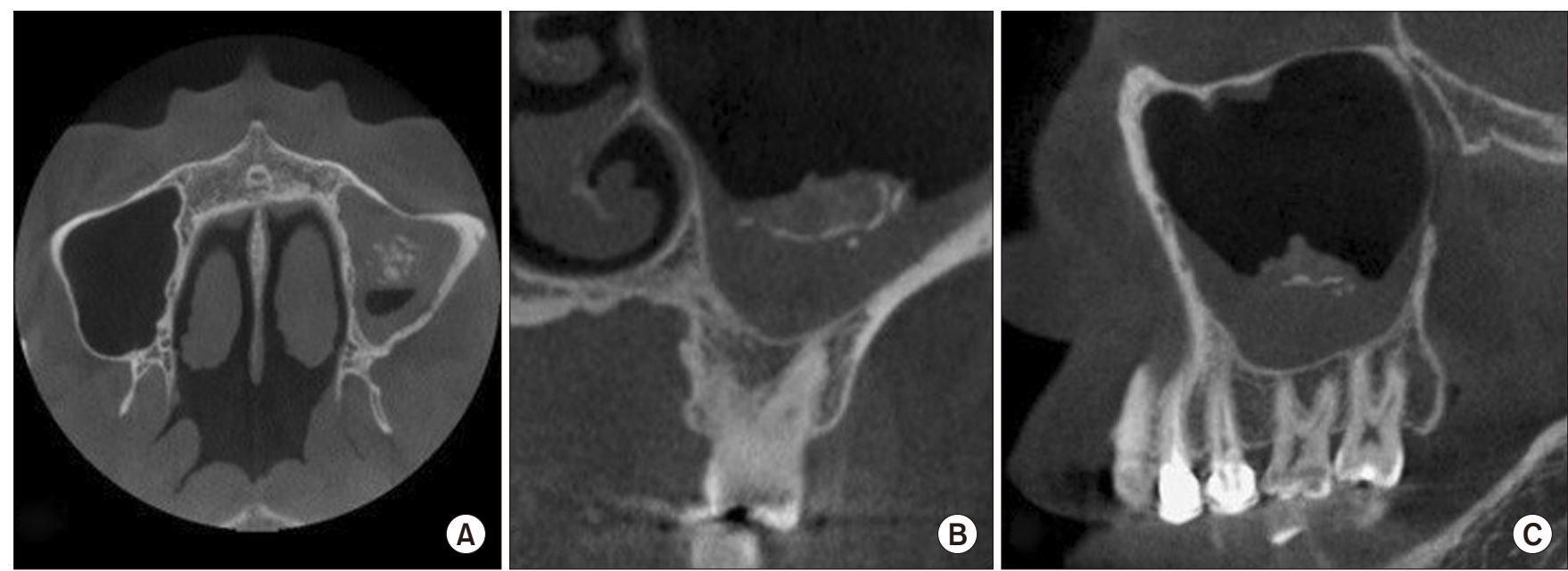

Fig. 2. Cone beam computed tomography view shows moderate mucosal thickening including small calcified bodies of the left maxillary sinus (A) Axial view, (B) coronal view, (C) sagittal view. 
upper dentition at a dental clinic. However, toothache was not disappeared. She referred to the department of conservative dentistry at Jeonbuk National University Hospital. Although panoramic and periapical radiograph showed no specific lesions and findings, CBCT showed fungal sinusitis in the right maxillary sinus (Fig. 3, 4). She was referred to the department of otorhinolaryngology for treatments of the right maxillary sinus. It was also diagnosed to FB infection from nasal endoscopic features and histopathological findings after surgical removal under general anesthesia (Fig. 5).

She visited our department about 3 weeks later after the surgery because of persisting toothache on the right upper molar area during mastication and uncomfortable sensation on the right middle face area.

Clinical examination showed palpation pain on both masseter muscles and first premolar, second premolar and first molar of right upper dentition sensitivity to percussion.

The patient was diagnosed as local myalgia and medication,

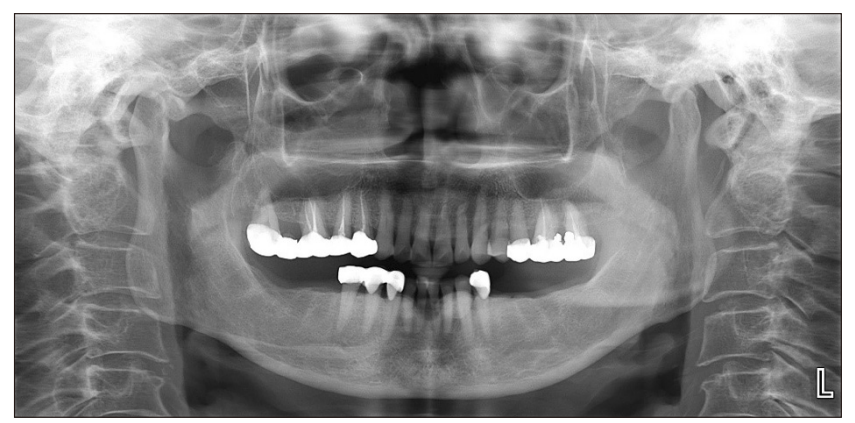

Fig. 3. Panoramic view shows no remarkable findings. physical therapy and patient education were given. We had planned to monitor the progress of the main symptoms over time. Her toothache got gradually declined two months later after the surgery.

\section{DISCUSSION}

FB of the paranasal sinuses is a non-invasive form of fungal sinusitis defined as the accumulation of dense conglomeration of fungal hyphae in sinus cavities [8,9].

Although it was considered relatively rare in the past, the number of patients has increased over the past two decades [9].

It occurs predominantly in older people and more frequently observed in females. The mean age of the patients

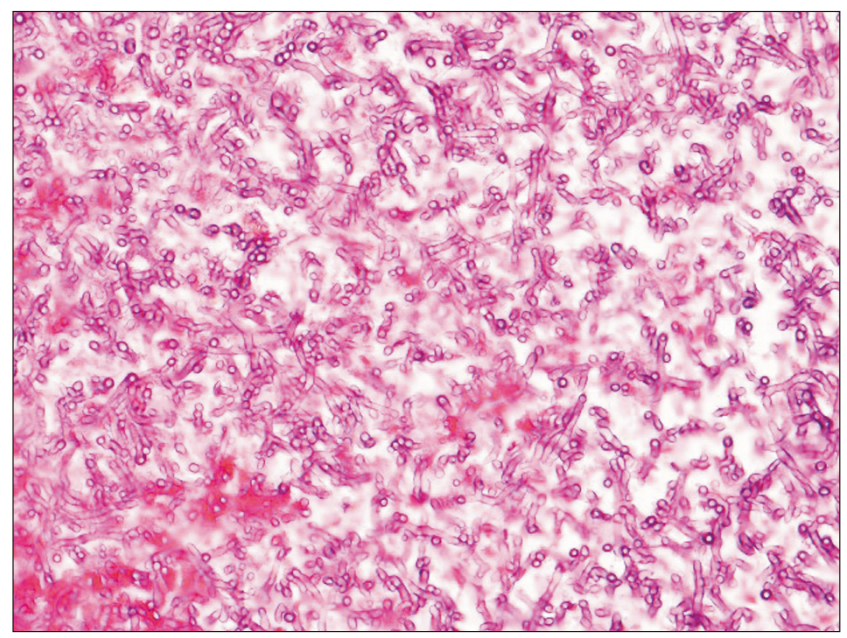

Fig. 5. Aggregates of highly packed hyphae are observed in microscopic examination (H\&E staining, $\times 400)$.
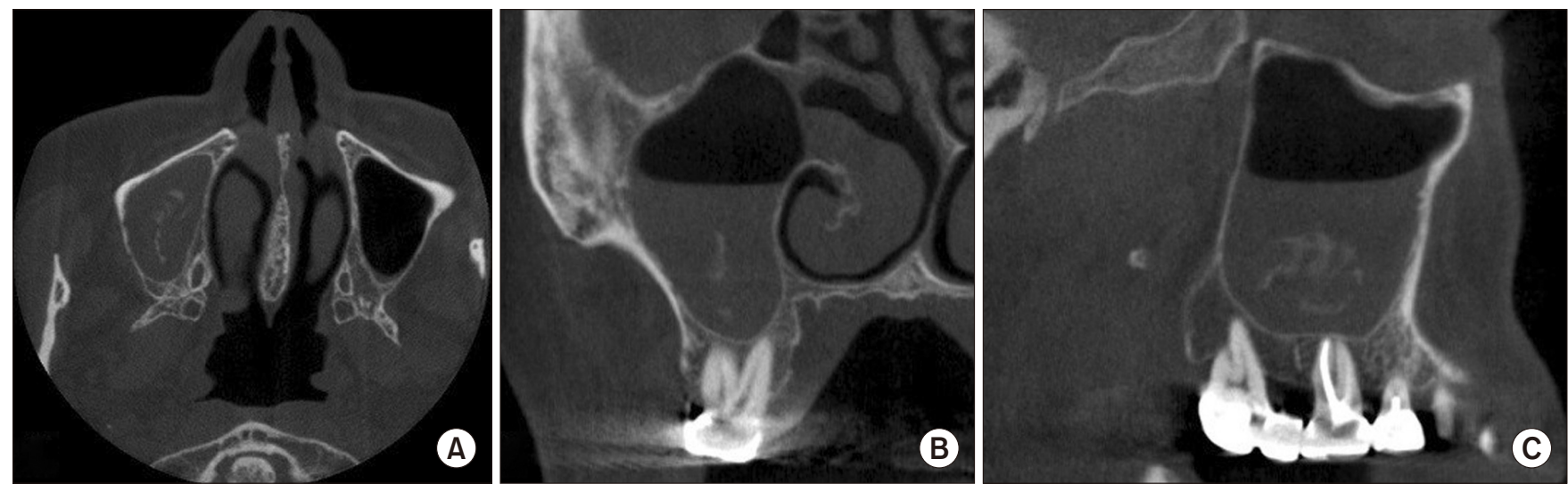

Fig. 4. Cone beam computed tomography view shows fungal sinusitis in the right maxillary sinus. (A) Axial view, (B) coronal view, (C) sagittal view. 
was 53.6 and the ratio of female patients was 62.5\% [10]. Klossek et al. [11] reported that most of FB can be found in a single sinus of patients. FB is most frequently occurred in the maxillary sinus followed by the sphenoid sinus [10].

The pathogenesis of FB remains unexplained. No predisposing environmental or local anatomical factors have been demonstrated although most of the patients are usually immunocompetent [8]. However, two pathways of fungal entry have been suggested. An aerogenic pathway theory explains that high quantities of the airborne spores can enter the sinus and turn to be pathogenic when the sinus became anaerobic. Another pathway of fungal colonization in which occurs in the maxillary sinus is the iatrogenic oroantral communication caused by dental extraction or root canal treatment [8].

FB is known to be asymptomatic in the early stage and developed slowly. It can be diagnosed incidentally in asymptomatic patients by computed tomography or magnetic resonance imaging for head examination in a part of the diagnosis of other diseases $[8,12]$. At the later stages of the disease, it causes non-specific clinical symptoms of chronic rhinosinusitis including post-nasal drip, nasal congestion, facial pain, headache, toothache, and retro-orbital pain [12].

In our cases, the patients complained of toothache in the upper molar area. Fungal sinusitis of maxillary sinus was incidentally found from CBCT images performed to find odontogenic causes.

The radiological findings of $\mathrm{FB}$ are quite specific. Heterogenic densities with focal dense calcification could be found in conventional tomography (CT). The calcified bodies of the FB observed at CT are caused by calcium deposits from metabolic reactions of fungal mass $[10,13]$.

Yoon et al. [14], reported that 21 of 39 patients with fungal sinusitis had intra-sinus calcifications, while 16 of 471 patients with nonfungal sinusitis had it. The location of calcification was mainly central of the maxillary sinus (95\%) in fungal sinusitis and the calcification had irregular margins with fine punctate, linear or nodular form. Smoothmargined calcification was only seen in nonfungal sinusitis [14].

Histopathological and mycological examinations are also needed to establish the diagnosis [13]. Microscopically, aggregates of highly packed hyphae can be observed and the sinus mucosa shows non-specific inflammation [8]. It has to be confirmed no invasion of the sinus mucosa or bone by fungi in order to have a positive diagnosis of the FB [13].

Fungal cultures show a relatively poor yield. However, it is recommended to perform in every case to identify the causative fungal species, as complementary to special staining techniques [8].

Functional endoscopic sinus surgery with a canine fossa approach is the treatment of choice [8,9]. Most patients may not need further antifungal treatment and rare recurrences had been reported $[8,15]$.

In these present cases, toothache was alleviated after FB removal surgery. As mentioned earlier, it could cause nonspecific clinical symptoms including facial pain, and toothache at the later stages of the disease [12].

Similar to cases we reported, Yoon et al. [9] reported that $6.1 \%$ of patients (29 out of 477) had toothache caused by the FB of the maxillary sinus. Ahn et al. [12] reported that toothache was found in 3.8\% of patients (1 out of 26) in the bilateral FB group and 3.9\% of patients (16 out of 408) in the unilateral FB group. Sinusitis caused by FB is thought to induce toothache $[8,9]$.

The exact mechanism of toothache caused by sinusitis was not fully understood, in so far. Toothache frequently occurs in the maxillary premolar and molar regions in acute sinusitis, whereas chronic sinusitis usually does not accompany toothache $[16,17]$. In contrary to general belief, chronic sinusitis seldom induces facial pain or headache. Sinus lining mucosa is known to be nearly insensible to pain. Referred spontaneous toothache does not arise as a result of sinus inflammation. A condition that causes toothache in sinusitis was suggested that neuritic neuralgia could occur by inflammation of superior alveolar plexus [18]. The neuritic symptoms occur in the peripheral distribution of the affected nerve branches because the dental nerve often lies just below the lining mucosa or are separated by a very thin bone. These conditions may cause pain in the ipsilateral maxillary teeth [18].

As we mentioned before, nonodontogenic toothache could be caused by a variety of causes, including fungal sinusitis of the maxillary sinus. Therefore, dental practitioners should consider the nonodontogenic toothache and avoid performing unnecessary dental treatment such as root canal 
treatment or extractions when clinical and radiographic findings did not reveal dental origin.

\section{CONFLICT OF INTEREST}

No potential conflict of interest relevant to this article was reported.

\section{ORCID}

\author{
Ji-Woo Ha \\ https://orcid.org/0000-0002-6708-796X \\ Won Jung \\ https://orcid.org/0000-0002-8728-8013 \\ Kyung-Eun Lee \\ https://orcid.org/0000-0001-8923-1478 \\ Bong-Jik Suh \\ https://orcid.org/0000-0002-1817-4645
}

\section{REFERENCES}

1. Soni A. Toothache of non-dental origin: a review of its mechanism and clinical characteristics. Int J Sci Study 2018;6:26-35.

2. Chung JW, Kim JH, Kim HD, Kho HS, Kim YK, Chung SC. Chronic orofacial pain among Korean elders: prevalence, and impact using the graded chronic pain scale. Pain 2004;112:164-170.

3. Balasubramaniam R, Turner LN, Fischer D, Klasser GD, Okeson JP. Non-odontogenic toothache revisited. Open J Stomatol 2011;1:92-102.

4. Kim KM, Byun JS, Jung JK, Choi JK. Non-odontogenic toothache caused by acute maxillary sinusitis: a case report. J Oral Med Pain 2016;41:80-84.

5. Kang JK, Ryu JW. Nonodontogenic toothache. Oral Biol Res
2018;42:241-247.

6. Mascia P, Brown BR, Friedman S. Toothache of nonodontogenic origin: a case report. J Endod 2003;29:608-610.

7. Linn J, Trantor I, Teo N, Thanigaivel R, Goss AN. The differential diagnosis of toothache from other orofacial pains in clinical practice. Aust Dent J 2007;52(1 Suppl):S100-S104.

8. Grosjean P, Weber R. Fungus balls of the paranasal sinuses: a review. Eur Arch Otorhinolaryngol 2007;264:461-470.

9. Yoon YH, Xu J, Park SK, Heo JH, Kim YM, Rha KS. A retrospective analysis of 538 sinonasal fungus ball cases treated at a single tertiary medical center in Korea (1996-2015). Int Forum Allergy Rhinol 2017;7:1070-1075.

10. Pinar E, Imre A, Ece AA, et al. Paranasal sinus fungus ball: analysis of clinical characteristics and surgical outcomes. ENT Updates 2015;5:124-127.

11. Klossek JM, Serrano E, Péloquin L, Percodani J, Fontanel JP, Pessey JJ. Functional endoscopic sinus surgery and 109 mycetomas of paranasal sinuses. Laryngoscope 1997;107:112-117.

12. Ahn SH, Lee EJ, Hong MP, Shin GC, Kim KS. Comparison of the clinical characteristics of bilateral and unilateral fungal balls in Korea. Eur Arch Otorhinolaryngol 2019;276:1975-1980.

13. Patrascu E, Manea C, Sarafoleanu C. Difficulties in the diagnosis of fungal rhinosinusitis - literature review. Rom J Rhinol 2016;6:11-17.

14. Yoon JH, Na DG, Byun HS, Koh YH, Chung SK, Dong HJ. Calcification in chronic maxillary sinusitis: comparison of CT findings with histopathologic results. AJNR Am J Neuroradiol 1999;20:571-574.

15. Aribandi M, McCoy VA, Bazan C 3rd. Imaging features of invasive and noninvasive fungal sinusitis: a review. Radiographics 2007;27:1283-1296.

16. Yatani H, Komiyama O, Matsuka Y, et al. Systematic review and recommendations for nonodontogenic toothache. J Oral Rehabil 2014;41:843-852.

17. Hansen JG, Højbjerg T, Rosborg J. Symptoms and signs in culture-proven acute maxillary sinusitis in a general practice population. APMIS 2009;117:724-729.

18. Okeson JP. Bell's oral and facial pain. 7th ed. Chicago: Quintessence Publishing Co, Inc; 2014. pp. 384, 458-459 